\title{
Visualizing Electron-Molecule Dynamics with In-situ Fluorescence in a Few eV- SEM
}

Y. $\operatorname{Vos}^{1 *}$, C.W. Hagen ${ }^{1}$, and J.P. Hoogenboom ${ }^{1 *}$

${ }^{1}$ Imaging Physics, Delft University of Technology, Lorentzweg 1, 2628CJ Delft, The Netherlands

* Corresponding authors: Y.Vos-1@tudelft.nl,j.p.hoogenboom@tudelft.nl

Understanding and directing the pathways of reactions between molecules and energetic electrons is important for a wide variety of technological applications such as electron microscopy, electron- and XUV-lithography, and electron-beam induced deposition. In most cases, reactions with low-energy secondary electrons (energies of $<50 \mathrm{eV}$ ) generated in the environment of the molecule, have cross sections dominating over contributions from higher energy electrons. For these low energy electrons, there can be many different degradation and/or dissociation pathways involved at the molecular level [1]. Disentangling the different contributions can be challenging, especially for complex but relevant samples such as polymers, organic compounds, and biological tissues. Typically, one evaluates in some experimental way the end products obtained after reaction, leaving transient dynamics obscured. Here, we present a novel approach to study low-energy electron induced molecular degradation by combining in-situ fluorescence microscopy with electron irradiation of organic fluorescent molecules to report on the reaction kinetics occurring in the sample.

We use an inverted fluorescence light microscope (Delmic Secom) integrated in a scanning electron microscope (SEM; FEI Verios 460 ) (Fig 1a) [2][3]. Electron beam landing energies are controlled to single eVs by applying a negative stage bias and using the SEMs monochromator (Fig. 1b). To demonstrate our approach, we used a sample of fluorescent perylene diimide molecules (PTDI) spincoated on a glass/ITO substrate that was coated with an $\mathrm{Al}_{2} \mathrm{O}_{3}$ layer of several nanometres.

The perylene molecules are exposed to fixed, low electron doses with landing energies ranging from 0 to $40 \mathrm{eV}$ while we monitor the perylene fluorescence intensity using continuous light illumination. Our results show an electron-energy dependent decrease in fluorescence after irradiation $\left(\mathrm{I}_{\mathrm{drop}}\right)$, followed by a fractional recovery of the fluorescence intensity ( $\mathrm{I}_{\text {recovery }}$ ) occurring over a timescale of several seconds (see Fig. 2). This timescale indicates that we not only visualize the processes of direct beam damage via $\mathrm{I}_{\mathrm{drop}}$, but also the effect of generated side products in the sample by looking at I recovery.

Comparing the energy dependence of the initial drop in fluorescence intensity with the subsequent fluorescence recovery gives insight in the relaxation processes occurring in the sample. A minimum in $\mathrm{I}_{\mathrm{drop}}$ is reached at an electron landing energy of $7 \mathrm{eV}$, which we describe by being the energy threshold for ionization processes (i.e. ejection of the electron from the molecule). Furthermore, a peak at $1 \mathrm{eV}$ for $\mathrm{I}_{\mathrm{drop}}$ is described by electron attachment to the molecule. A rise in the fractional recovery $\mathrm{I}_{\text {recovery }} / \mathrm{I}_{\text {drop }}$ can be found around $10 \mathrm{eV}$. The fluorescence recovery will be discussed by radical (di)anion formation and subsequent quenching of the fluorescence and electron charge transport. 
Combining light- and electron microscopy enables visualizing electron-molecule interactions by reducing the electron beam energy to few eVs. By using fluorescent molecules as a probe for chemical changes this not only allows to determine the permanent damage done to the sample as a function of energy, but can also show the energy-dependent dynamics incurred in the sample directly after irradiation by detection of recovery in the fluorescence intensity. This illustrates how our approach with beam deceleration techniques can be used to disentangle the energetic contributions of electron irradiation and to visualize the further course of relaxation in the sample.

[1] Van Dorp, Willem F. "Theory: electron-induced chemistry." Frontiers of Nanoscience. Elsevier Vol. 11 (2016), p. 115-133.

[2] Liv, Nalan, et al. "Simultaneous correlative scanning electron and high-NA fluorescence microscopy." PloS one 8.2 (2013), p. e55707.

[3] Zonnevylle, A. C., et al. "Integration of a high-NA light microscope in a scanning electron microscope." Journal of microscopy 252.1 (2013), p. 58-70.

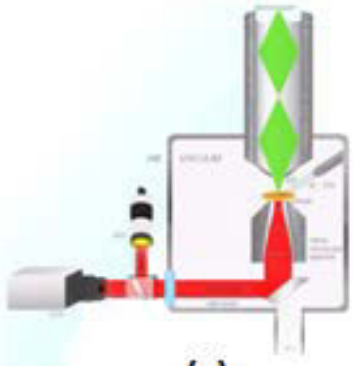

(a)

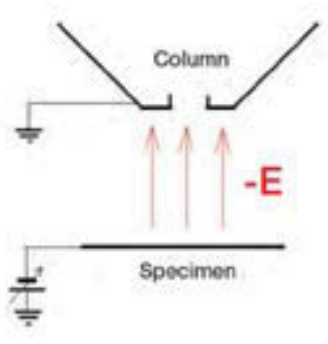

(b)

Figure 1. (a) Schematic for integrated microscopy, fluorescence microscopy (optical path in red) can be performed simultaneously with scanning electron microscopy (optical path in green). (b) Electron landing energies are controlled with a negative stage bias, decelerating electrons leaving the column.

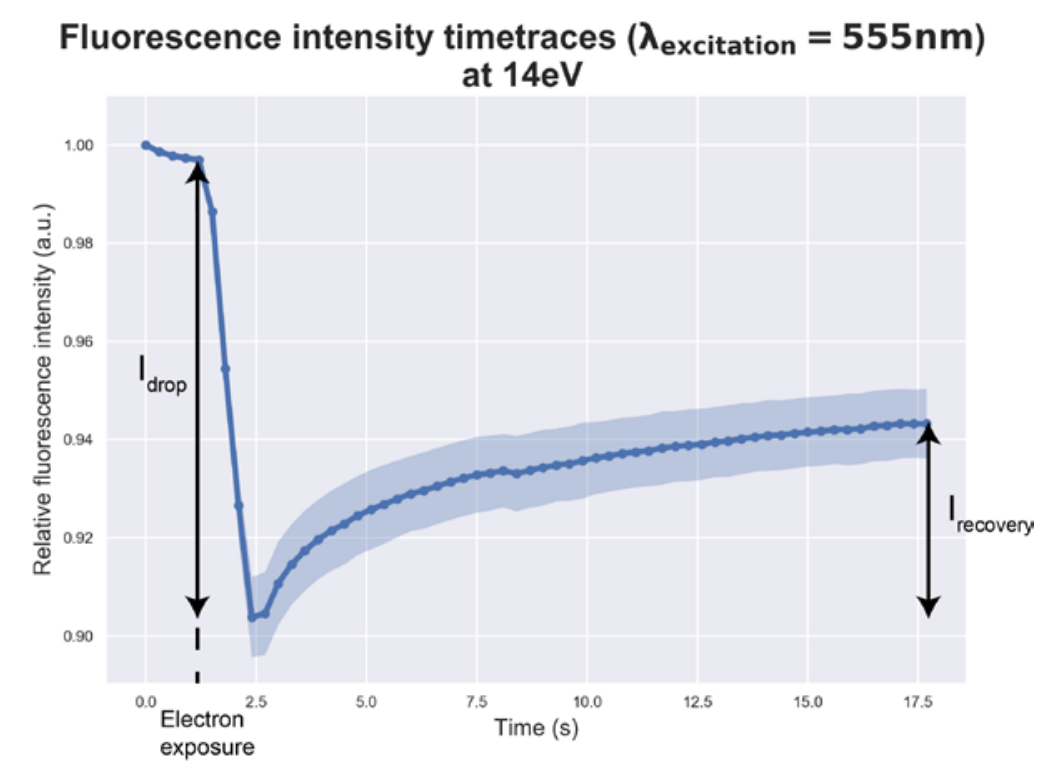

Figure 2. Fluorescence intensity curve with e-beam irradiation under continuous illumination. A strong drop in intensity $\left(\mathbf{I}_{\mathbf{d r o p}}\right)$ at $1.2 \mathrm{~s}$ corresponds to electron beam exposure with a dose of $\mathrm{D}=\mathbf{2 6} \mathbf{~ \mathbf { C C }} / \mathbf{m}^{-\mathbf{2}}$ and landing energy of $14 \mathrm{eV}$. A fractional recovery (I $\mathbf{I}_{\text {recovery }}$ ) occurring over a seconds timescale is observed. 